\title{
Speed Control of a Permanent Magnet Synchronous Machine Powered by an Inverter Voltage Moment Approach
}

\author{
J. Khedri, M. Chaabane, M. Souissi
}

\author{
Jamel Khedri, Mohamed Chaabane (corresponding author), Mansour Souissi \\ Research Unit of Industrial Processes Control \\ National School of Engineers of Sfax \\ ENIS, Route soukra, km 3.5 - BP.W, 3038, Sfax, Tunisia \\ E-mail: \{khedrijamel,chaabane_uca\}@yahoo.fr, mansour.souissi@ipeis.rnu.tn
}

\begin{abstract}
In this paper, the method of moments is presented in order to synthesize controllers for current and speed of a Magnet Permanent Synchronous Machine (PMSM). The controller's dynamics are chosen in respect with the time domain constraints, which have equality relationships between the moments of the closed-loop system and those of the reference model. The Linear Matrix Inequality (LMI) formalism is used for the controller synthesis. The proposed controller is applied for the speed tracking of a PMSM to illustrate the performances of the method.

Keywords: Times Specifications, method of moments, Reference Model, LMI, PMSM.
\end{abstract}

\section{Introduction}

New industrial applications require variable speed drives with high dynamic performance. In recent years several techniques of control have been developed, allowing PMSM with variable speed to achieve these performances. However, vector control, which allows decoupling between control variables remains the most widely used [1-3]. The main advantages of this configuration are that the regulatory cascade is a very industrial responded [4,5]. It gives high dynamic performance for a wide range of applications. The complexity of the dynamic model of the permanent magnet synchronous variable speed and the presence of external disturbances and parametric variations limit the performance of the control law [6]. Thus, our main contribution in this paper consist in introducing a time constraint with the help of a reference model, simple to operate and which characterizes the dynamics of the closed loop system. This time constraint is formulated by the equality of the first time moments of the closed-loop system and those of the reference model [7-11].

Practically, a first LMI is used to insure closed-loop stability, a second one permits, by minimizing the norm 2 of time moments cost, to identify time specifications (overshoot, response time ...).

Without loss of generality, the methodology has been restricted in this paper to the case of square, invertible, MIMO (multi inputs, multi outputs) systems.

The paper is organised as follows: in section 2, we define the state space representations of the systems and those of the controller; moreover, the problem of the design of a controller verifying stability and time performances is stated. In section 3, we propose a solution of this problem, owing to the demonstration of a specific theorem. Experimental results applied to a permanent magnet synchronous machine (PMSM) are presented in section 4 . 


\section{Problem statement}

Consider a Linear Time Invariant (LTI) square invertible system $H(s)$ with $m$ inputs and $m$ outputs:

$$
\left\{\begin{array}{c}
\dot{x}_{\text {sys }}(t)=A_{\text {sys }} x_{c}(t)+B_{\text {sys }} u(t) \\
y(t)=C_{\text {sys }} x(t)
\end{array}\right.
$$

The controller is modeled in the state space by:

$$
\left\{\begin{array}{c}
\dot{x}_{c}(t)=A_{c} x_{c}(t)+B_{c} e(t) \\
u(t)=C_{c} x_{c}(t)+D_{c} e(t)
\end{array}\right.
$$

with

$$
e(t)=r(t)-y(t)
$$

where: $x_{\text {sys }}(t) \in \mathbb{R}^{l}$ is the state vector of the system, $u(t) \in \mathbb{R}^{m}$ is the control input, $y(t) \in \mathbb{R}^{m}$ is the output, $r(t) \in \mathbb{R}^{m}$ is the reference of closed-loop system, Figure 1 . Matrices $A_{\text {sys }}, B_{\text {sys }}, C_{\text {sys }}$ are supposed to be known with appropriate dimensions. The controller model matrices $A_{c}, B_{c}$, $C_{c}$ and $D_{c}$ are to be computed.

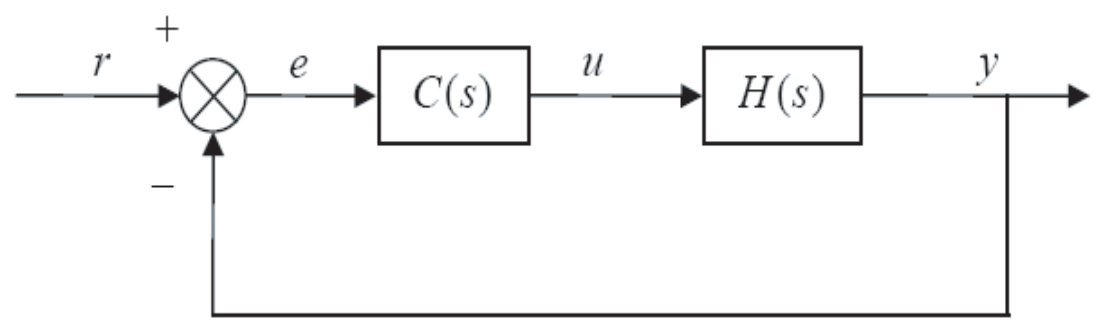

Figure 1: Closed-Loop System

The synthesis of controller requires the construction of the augmented model which regroups the system and the controller model. Consider the augmented state vector $z(t)$ defined as:

$$
z(t)=\left[\begin{array}{c}
x_{\text {sys }}(t) \\
x_{c}(t)
\end{array}\right]
$$

Then, the closed-loop system, Figure 1, can be represented by the following model:

$$
\left\{\begin{array}{c}
\dot{z}(t)=A_{b f} z(t)+B_{b f} r(t) \\
y(t)=C_{b f} z(t)
\end{array}\right.
$$

with:

$$
A_{b f}=\left[\begin{array}{cc}
A_{s y s}-B_{s y s} D_{c} C_{s y s} & B_{s y s} C_{c} \\
-B_{c} C_{s y s} & A_{c}
\end{array}\right] ; \quad B_{b f}=\left[\begin{array}{c}
B_{s y s} D_{c} \\
B_{c}
\end{array}\right] ; \quad C_{b f}=\left[\begin{array}{cc}
C_{s y s} & 0
\end{array}\right]
$$

Let's note that the closed-loop stability is ensured if and only if there exist a positive definite matrix $P$ with $P=P^{T}$, such as the LMI

$$
P A_{b f}+A_{b f}^{T} P<0
$$


is satisfied.

To facilitate the study, our work is restricted to the case where the controller $C(s)$ is to be synthesised. The controller design can be considered as a static feedback gain $K$ for the augmented system.

The controller synthesis is ensured by the fact that matrices $A_{c}$ and $B_{c}$ are defined a priori and only the matrices $C_{c}$ and $D_{c}$ are to be computed. For this purpose, we consider the following hypothesis:

The matrix $A_{b f}$ of augmented system can be written as follows:

$$
A_{b f}=\tilde{A}+\tilde{B} K \tilde{C}
$$

where:

$$
\tilde{A}=\left[\begin{array}{cc}
A_{\text {sys }} & 0 \\
-B_{c} C_{\text {sys }} & A_{c}
\end{array}\right] ; \quad \tilde{B}=\left[\begin{array}{c}
B_{\text {sys }} \\
0
\end{array}\right] ; \quad \tilde{C}=\left[\begin{array}{cc}
-C_{\text {sys }} & 0 \\
0 & I
\end{array}\right]
$$

It is to notice that the gain $K$ is defined as:

$$
K=\left[\begin{array}{ll}
D_{c} & C_{c}
\end{array}\right]
$$

The extended system is then characterized by $(\tilde{A}, \tilde{B}, \tilde{C})$. The objective of this work is to ensure closed-loop stability and to specify transient performances which are characterized by a reference model $T_{r e f}(S)$. The Dynamics of the reference model are determined by the use of the time moments approach. In this case, the time moments of transfer $W(S)$, which are represented in state space by $\left(A_{W}, B_{W}, C_{W}, D_{W}\right)$ (see [4]), are given by the following relations:

$$
\begin{gathered}
M_{W, 0}=-C_{W} A_{W}^{-1} B_{W}+D_{W} \\
M_{W, j}=(-1)^{j+1} C_{W} A_{W}^{-(j+1)} B_{W}+D_{W}
\end{gathered}
$$

$$
(j=1 \ldots \infty)
$$

$M_{W, 0}$ : is the $0^{t h}$ order moment.

The exposing $j$ refers to the order of moments. For applying the proposed moment approach, we will define briefly the first three time moments.

The $0^{t h}$ order moment $M_{0}$ is equal to the static gain of the system and represents the area of its impulse response. The first order moment $M_{1}$ characterizes the average time $t_{m}$, the same as the average value of a probability density: $t m=\frac{M_{1}(g)}{M_{0}(g)}$

$g(t)$ is the impulse response of the system, represented by Figure 2 .

For a first order system, we have $M_{1}=\tau M_{0}$ where represents the time constant of the system. More generally, $M_{1}$ is used to characterize the time response of the considered system.

The second order moment $M_{2}$, characterizes the variance $\sigma$ (or dispersion $\Delta \tau$ ) of the impulse response around its mean time $t m$.

In practice, the time moments $M_{0}, M_{1}, M_{2}$ carry sufficient information on the impulse response: static gain, mean time and dispersion. 


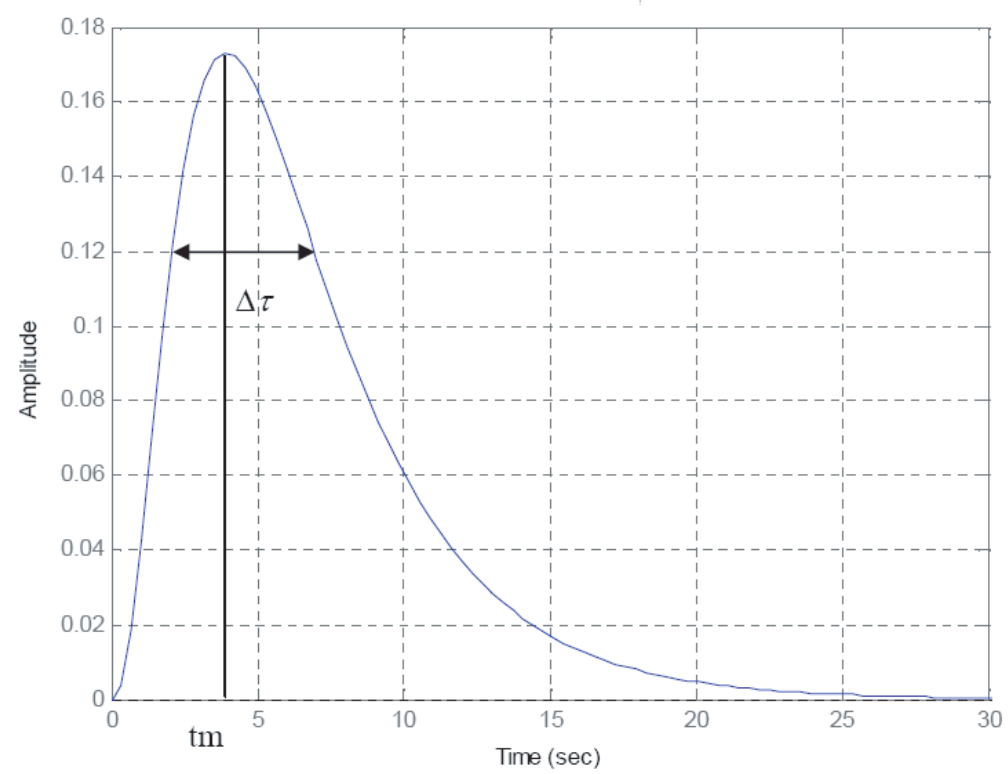

Figure 2: Characterization of an impulse response

The controller has to be designed such that the closed-loop transfer $T(S)$ is equal to the reference model $T_{r e f}(s)$.

$$
(I+H(s) C(s))^{-1} H(s) C(S)=T_{r e f}(s)
$$

or

$$
C(s)\left(I-T_{\text {ref }}(s)\right)=H^{-1}(s) T_{\text {ref }}(s)
$$

It is known that such control law $u(t)$ is in the form: $u(t)=K e(t)$ Using state space representation of $C(s)$, we can write:

$$
u(s)=\left[C_{c}\left(s I-A_{c}\right)^{-1} B_{c}+D_{c}\right] e(s)
$$

While referring to considered hypothesis given by relation (9), equation (13) can be written as:

$$
K\left[\begin{array}{c}
I \\
\left(s I-A_{c}\right)^{-1} B_{c}
\end{array}\right]\left(I-T_{r e f}(s)\right)=H^{-1}(s) T_{r e f}(s)
$$

If we set:

$$
\begin{gathered}
F(s)=H^{-1}(s) T_{r e f}(s) \\
W(s)=\left[\begin{array}{c}
I \\
\left(s I-A_{c}\right)^{-1} B_{c}
\end{array}\right]\left(I-T_{r e f}(s)\right)
\end{gathered}
$$

Then, the time constraint (15) can be expressed by:

$$
K W(s)=F(s)
$$

Notice that the last relation (18) is linear, then the formalism LMI can be applied. In fact, an ideal equality in the form (18) between the closed-loop $T(s)$ and $T_{r e f}(s)$ is not possible to obtain. 
Therefore, it is recommended to replace an ideal equality by an approximation between $T(s)$ and $T_{r e f}(s)$. In order to achieve this condition, we use the time moments approach, as mentioned previously.

Therefore, when the objective is to characterize the time response, the equality (18) is transformed to a minimization of the quadratic distance between the first $(n+1)$ time moments of the closed-loop system and its reference model, where the minimization criterion is defined as:

$$
\left(K M_{W, j}-M_{F, j}\right)^{T}\left(K M_{W, j}-M_{F, j}\right)<\gamma I
$$

$j=0,1, \ldots, n$.

Using this criterion, we can notice that the transient responses of $T(s)$ and $T_{r e f}(s)$ become approximately similar (they have approximately the same overshoot, the same response time, etc.)

The overall objective is achieved if there exist a symmetric and positive defined matrix $P$ and a gain $K$ given by relation (10). In such case, the closed-loop stability condition (7) and the minimization criterion condition (19) are satisfied. In the next section, we present a solution to this problem, which can be formulated by the theorem 1 .

\section{Mains results}

The objective of this section is to give solution of the previous problem.

\subsection{Theorem 1.}

Consider a LTI system $\left(A_{\text {sys }}, B_{\text {sys }}, C_{\text {sys }}\right)(1)$ and the corresponding augmented system $(\tilde{A}, \tilde{B}, \tilde{C})$ defined in (9). Consider a static state feedback $K_{e}$ stabilizing the pair $(\tilde{A}, \tilde{B})$.

The stability of the closed-loop system and the time constraints imposed by the reference model $T_{r e f}(s)$ are guaranteed, if the optimisation problem:

Minimum $\gamma$

$(L, G, P)$ with the constraints

$P>0$

$$
\left[\begin{array}{cc}
\operatorname{Sym}\left\{P\left(\tilde{A}+\tilde{B} K_{e}\right)\right\} & \left(L \tilde{C}-G K_{e}\right)^{T}+P \tilde{B} \\
\left(L \tilde{C}-G K_{e}\right)+\tilde{B}^{T} P & -\left(G+G^{T}\right)
\end{array}\right]<0
$$

and

$$
\left[\begin{array}{cc}
-I & \left(L M_{W, j}-G M_{F, j}\right) \\
\left(L M_{W, j}-G M_{F, j}\right)^{T} & -\gamma I
\end{array}\right]<0 j=0,1, \ldots, n .
$$

has a solution, then the gain of dynamic controller $C(s)$ is given by :

$$
K=G_{o p t}^{-1} L_{o p t}
$$

Notice that $L_{\text {opt }}, G_{\text {opt }}$ correspond to the minimal value of $\gamma$ denoted $\gamma_{o p t}$. Before demonstration of Theorem 1, let us recall the following result $[6,7]$.

\subsection{Lemma 1.}

Consider the augmented system (5), where the state matrix $A_{b f}$ is defined by (8). The following statements are equivalent: 
1) There exist a symmetric and positive defined matrix $P=P^{T}>0$ and two matrices $K_{e}$ and $K$ such that:

$$
\begin{gathered}
P\left(\tilde{A}+\tilde{B} K_{e}\right)+\left(\tilde{A}+\tilde{B} K_{e}\right)^{T} P<0 \\
P(\tilde{A}+\tilde{B} K \tilde{C})+(\tilde{A}+\tilde{B} K \tilde{C})^{T} P<0
\end{gathered}
$$

2) There exist a symmetric and positive defined matrix $P=P^{T}>0$, a non singular matrix $G$ and two matrices $K_{e}$ and $K$ such that:

$$
\left[\begin{array}{cc}
\operatorname{Sym}\left\{\left(\tilde{A}+\tilde{B} K_{e}\right)^{T} P\right\} & P \tilde{B} \\
\tilde{B}^{T} P & 0
\end{array}\right]+\operatorname{sym}\left\{\left[\begin{array}{c}
0 \\
I
\end{array}\right] G\left[\left(K \tilde{C}-K_{e}\right)-I\right]\right\}
$$

\subsection{Proof of lemma 1}

First of all, notice that the orthogonal complements of the second term of (25) are: $V=\left[\begin{array}{ll}I & 0\end{array}\right]$, the orthogonal complement of $V$, such that $V V^{\perp}=0$, is $V^{\perp}=\left[\begin{array}{l}0 \\ I\end{array}\right]$; and $U=\left[\begin{array}{ll}I & \left(K \tilde{C}-K_{e}\right)^{T}\end{array}\right]$, the orthogonal complement of $U$, such that $U U^{\perp}=0$, is: $U^{\perp}=\left[\begin{array}{c}\left(K \tilde{C}-K_{e}\right)^{T} \\ -I\end{array}\right]$

Applications of elimination lemma [9] lead to left and right multiplication of (25) by $V$ and $V^{\perp}$ respectively, which gives inequality (23):

$$
\left[\begin{array}{ll}
I & 0
\end{array}\right] \operatorname{sym}\left\{\left[\begin{array}{l}
0 \\
I
\end{array}\right] G\left[\left(K \tilde{C}-K_{e}\right)-I\right]\right\}\left[\begin{array}{l}
I \\
0
\end{array}\right]=P\left(\tilde{A}+\tilde{B} K_{e}\right)+\left(\tilde{A}+\tilde{B} K_{e}\right)^{T} P<0
$$

Even, if (25) is left and right multiplied respectively by $U$ and $U^{\perp}$, we get inequality (24).

\subsection{Proof of Theorem 1}

By using Shur complement, the condition (21) can be expressed as:

$$
\left(L M_{W, j}-G M_{F, j}\right)\left(L M_{W, j}-G M_{F, j}\right)^{T}<\gamma I
$$

$j=0,1, \ldots, n$.

Besides, if one multiplies equation (18) by $G$ :

$$
L W(s)=G F(s)
$$

equation (19) becomes:

$$
\left(L M_{W, j}-G M_{F, j}\right)\left(L M_{W, j}-G M_{F, j}\right)^{T}<\gamma I
$$

$j=0,1, \ldots, n$.

One demonstrates that (26) and then the condition (21) correspond to the minimization of the 2 norm of the error between the $(n+1)$ first moments of the closed-loop and those of the reference model. If the minimization of $\gamma_{o p t}$ is satisfied, then the two transfers $T(s)$ and $T_{r e f}(s)$ are very close in low frequencies, which implies the desired transients for the closed-loop system. The stability is also insured due to the fact that condition (20) implies condition (24), equivalent to condition (7) which expresses closed-loop stability. 


\section{Application to the Permanent Magnet Synchronous Machine (PMSM)}

Our Permanent Magnet Synchronous Machine (PMSM) is powered by a two levels voltage inverter. To obtain non reciprocating quantities, our model is done in the Park landmark [10]. Thus we have the following equations in the synchronously d-q reference frame $[11,12]$ :

$$
\left\{\begin{array}{c}
\dot{I}_{d}=\frac{-R_{s}}{L_{d}} I_{d}+\frac{L_{q}}{L_{d}} w_{r} I_{q}+\frac{1}{L_{d}} v_{d} \\
\dot{I}_{q}=\frac{-R_{s}}{L_{q}} I_{q}-\frac{L_{d}}{L_{q}} w_{r} I_{d}-\frac{\phi}{L_{q}} w_{r}+\frac{1}{L_{q}} v_{q} \\
\dot{w}_{r}=p \frac{\left(c_{e}-c_{r}\right)}{J}-\frac{f_{c}}{J} w_{r} \\
c_{e}=p\left[\left(L_{d}-L q\right) I_{d}+\phi\right] I_{q}-\frac{f_{c}}{J} w_{r}-\frac{f_{c}}{J} w_{r} \\
\dot{w}_{r}=\frac{p^{2}}{J}\left[\left(L_{d}-L q\right) I_{d}+\phi\right] I_{q}-\frac{f_{c}}{J} w_{r}-\frac{p}{J} c_{r}
\end{array}\right.
$$

where $v_{d}$ and $v_{q}$ are the stator voltages, $I_{d}$ and $I_{q}$ are the stator currents, $L_{d}$ and $L_{q}$ are the inductances, $R_{s}$ is the stator winding resistance, $\phi$ is the flux linkage of the permanent magnets, $w_{r}$ is the angular velocity of the motor shaft, $f_{c}$ is the friction coefficient relating to the rotor speed; $J$ is the moment of inertia of the rotor,$c_{e}$ is the electromagnetic torque, $c_{r}$ is the load torque and $p$ is the number of pole. In an equivalent manner, these equations can be written in the form of a general transfer function $H(s)$ who contains the open-loop transfer of variables to control namely $H_{i d}(s)$ and $H_{w r}(s)$.

$$
H(s)=\left[\begin{array}{cc}
H_{i d}(s) & 0 \\
0 & H_{w r}(s)
\end{array}\right]
$$

where:

$$
H_{i d}(s)=\frac{\frac{1}{R_{s}}}{1+\tau_{i d}(s)}, \quad H_{w r}(s)=\frac{H_{b f I q} \frac{p^{2} \phi}{f_{c}}}{1+\tau(s)}
$$

where $\tau_{i d}=\frac{L_{d}}{R_{s}} ; \tau=\frac{J}{f_{c}}$

Let $H_{b f I q}(s)$,is the closed-loop transfer of the current $I_{q}: H_{b f I q}=\frac{1}{1+\tau_{b f i q}(s)} \tau_{b f i q}$ is chosen equal to $7.017510-4 \mathrm{~s}$ in order to perform a time response of the closed-loop system ten times faster than open-loop.

Notice that: $\tau_{i q}=\frac{L_{q}}{R_{s}} ; H_{i q}(s)=\frac{\frac{1}{R_{s}}}{1+\tau_{i q}(s)}$

The objective is to reduce the time response of the system, to keep the overshoot of the closedloop inferior to $5 \%$ while decoupling the two outputs. These specifications can be carried out by a second order reference model with static unity gain for the speed, and a first order one for the current $I_{d}$ :

$$
T_{\text {ref }}(s)=\left[\begin{array}{cc}
\frac{1}{\left.1+\tau_{\text {idref }} s\right)} & 0 \\
0 & \frac{1}{1+\frac{2 \zeta}{w}(s)+\frac{s^{2}}{w^{2}}}
\end{array}\right]
$$

The design choices correspond to $\tau_{i d r e f}=0.1 \tau_{i d}$ in order to perform a time response of the closed-loop current ten times faster than the open-loop.

$\zeta=7.6205, w=93.906 r d s-1$ in order to obtain a closed-loop speed 3.3 times faster than the open-loop one. Let's note that $H_{w r}$ has two constants time:

$\tau=\frac{J}{f_{c}}=0.5333 s, \tau_{1}=7.047210^{-4} s^{-1}$ The two constants time of the closed-loop speed are $\tau b f=0.1618 s, \tau_{1 b f}=7.017510^{-4} s$ Notice that the decoupling specification is insured by a diagonal reference model. The $\mathrm{d}$-axis current $I d$ is regulated to follow a zero setpoint. The static 
state feedback $K_{e}$ stabilizing the pair $(\tilde{A}, \tilde{B})$ such as $(23)$ feasible has been computed:

$$
K_{e}=\left[\begin{array}{ccccc}
-79.1667 & 0 & 0 & 722 & 0 \\
0 & 0 & -2.1433 & 0 & 0.0939
\end{array}\right]
$$

Solving LMIs (20) and (21) by LMI optimization algorithm, the matrices $P, G$ and $L$ can be obtained:

$$
G=10^{8}\left[\begin{array}{cc}
0.0065 & 0 \\
0 & 4.5881
\end{array}\right] ; \gamma_{o p t}=6.609610^{-14}
$$

The obtained static gain is:

$$
K=\left[\begin{array}{cccc}
5.7 & 0 & 722 & 0 \\
0 & 0.5006 & 0 & 0.09386
\end{array}\right]
$$

\section{Experimental results and interpretations}

The implementation of our model was performed on a test benchmark consisting of two Permanent Magnet Synchronous Machines (figure 3) fitted by the same encoder and powered by a voltage inverter; one was used as a motor and the other one as a load. The proposed control algorithm was executed by the use of Matlab/ Simulink software. Then it was compiled and implemented on Dspace 1104. The digital sampling period was taken equal to $0.1 \mathrm{~ms}$.


Figure 3: Photo of the test bench. 
Figures (4)-(6) illustrate the experimental results of the dynamic behaviour of PMSM for speed operating without charge.

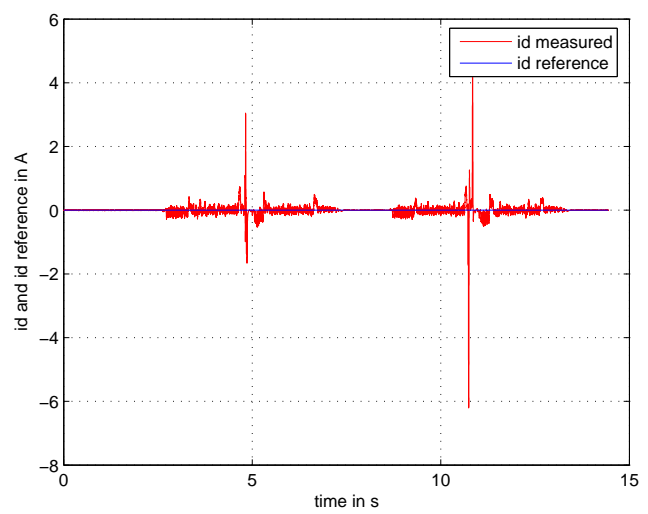

Figure 4: d-axis current and its reference

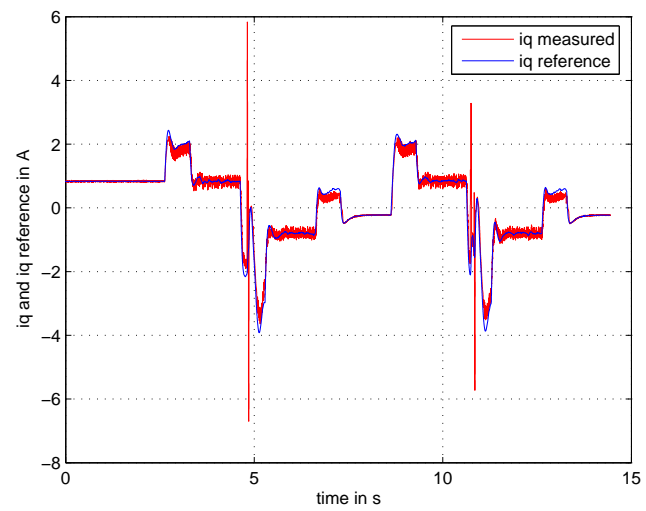

Figure 5: q-axis current and its reference

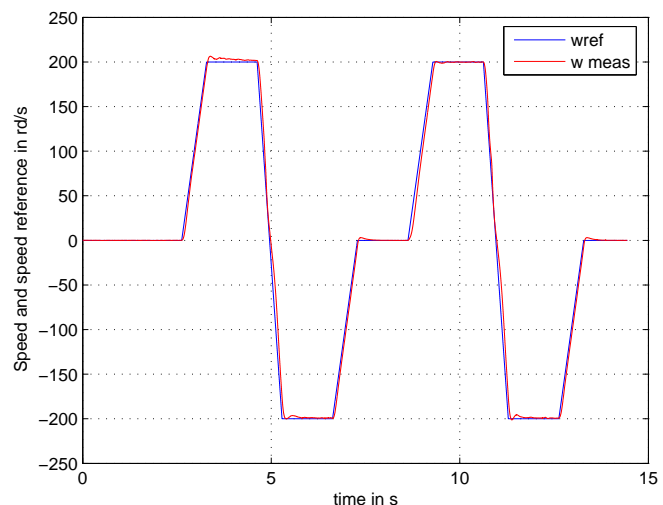

Figure 6: Speed and its reference

Whose data are listed in the Appendix. The results show that the closed loop system with 
the synthesized controller have a good behaviour: indeed, the measured speed and dq-axis currents track well the trajectory of reference one with good accuracy over the whole speed range, moreover, the time constraint imposed with the help of time moments has permitted to keep the closed loop outputs very close to those of the reference model.

\section{Conclusion}

In this paper, a controller design, for current and speed tracking of a PMSM has been developed by using the method of moments. The controller's dynamics are chosen in respect with the time domain constraints. These constraints have equality relationships between the moments of the closed-loop system and those of the reference model. The proposed controller has been achieved in order to ensure the closed-loop stability and to specify transient performances in regard with a reference model. In this study, the LMI formalism has been used for the controller synthesis. Finally, we have applied the proposed controller for the speed tracking of a PMSM which has given satisfactory results.

\section{Acknowledgements}

The authors wish to thank all the team of the Laboratory of Automatic and Industrial Informatics of the University of Poitiers FRANCE and special thanks to Pr. Gerard Champenois for the aid he afforded us for the realization of our experimental work validation.

\section{Appendix : Motor parameters}

\begin{tabular}{|c|c|}
\hline Motor rated power & $1 \mathrm{KW}$ \\
\hline Rated current & $6.5 \mathrm{~A}$ \\
\hline Pole pair number $(\mathrm{p})$ & 2 \\
\hline d-axis inductance Ld & $4.5 \mathrm{mH}$ \\
\hline Stator resistance & $0.56 \Omega$ \\
\hline Motor inertia J & $2.08 .10-3{\mathrm{Kg} . \mathrm{m}^{2}}^{-1}$ \\
\hline Friction coefficient fc & $3.9 .10^{-3} \mathrm{Nm} . \mathrm{s} . \mathrm{rad}^{-1}$ \\
\hline Magnet flux constant $\phi$ & $0.064 \mathrm{wb}$ \\
\hline
\end{tabular}

\section{Bibliography}

[1] Vas, P., Sensorless vector and direct torque control, Oxford, U. K, Oxford Univ. Press, 1998.

[2] Bose, B. K., Power electronics and AC drives, Englewood Cliffs, NJ, Prentice-Hall,1986.

[3] Bose, B. K., Power electronics and variable frequency Drives, Technology and Appliquation,IEEE press 1997.

[4] Flaus, J. M, Industrial control, Hermes, Paris 1994.

[5] Maret, L., automatic control, Polytechnic Press romandes, 1987. 
[6] Lin, F-J, Real time position controller design with torque feedforward control for a PM synchronous motor, IEEE Trans. On Industrial Electronics, Vol.44, $\mathrm{N}^{\circ} .3$, June 1997, pp. 398-407.

[7] Bentayeb, A., N. Maamri and J-C Trigeassou, The Moments in Control: a tool for Analysis, Reduction and Design, International Journal of Computers, Communications $\&$ Control (IJCCC). Vol. 2, num. 1, pp. 82-103, 2007.

[8] Maamri, N. and Trigeassou, J. C, PID design for time delayed systems by the method of moments ,European Control Conference, Groningen Holland (1993).

[9] Maamri, N. and Trigeassou, J. C, Controllers design by moments placement ,European Control Conference, Rome Italy (1995).

[10] Maamri, N., Bentayeb, A. and Trigeassou, J. C, Design and Iterative optimization of Reduced Robust Controllers with Equality Constraints, RECOND-Milan (2003).

[11] Trigeassou, J. C., Method of moments in automatic . Conférence Internationale Francophone d'Automatique, CIFA, Lille (2000).

[12] Mehdi, D., Control theory some insights . Report Automatic Control UCA-ENIS Sfax Tunisia Avril (2004).

[13] Peaucelle, D. and Arzelier, D., An efficient numerical solution for H2 static output feedback synthesis . European Control Conference, 2001, pp.3800-3805.

[14] Skelton, R. E., Iwasaki, T. and Grigoriadis, K., a unified algebraic approach to linear control design, Taylor \& Francis, (1998).

[15] Bose, B. K., Power electronics and AC drive . New york, Prince Hall, first Edition, (1986), chapter 2, page 95 .

[16] Sturtzer, G. and Sanigiel, E., Modeling and Control of three-phase motors .

[17] Hassaine, S., Applications of new technology command to permanent magnet synchronous machine. Thesis, ESIP, Poitiers, March 2008. 